\title{
GEOMETRICAL ASPECTS OF PARACONTACT PAIR STRUCTURES
}

\author{
ADARA M. BLAGA
}

Communicated by Izu Vaisman

\begin{abstract}
We introduce the notion of (metric) paracontact pair structure and establish certain properties of the characteristic foliations associated to it. We also consider normal paracontact pair structures and provide necessary and sufficient conditions for a paracontact pair structure to be normal. In particular, we formulate an analogue of Morimoto's theorem for product manifolds. Finally, we describe a way to obtain a metric paracontact pair structure on the total space of a principal $\mathbb{S}^{1}$-bundle via the Boothby-Wang construction.
\end{abstract}

\section{Introduction}

The contact pairs were defined by Blair, Ludden and Yano [10] under the name of bicontact. Further they were studied by Bande and Hadjar [3,4], Bande, Ghiggini and Kotschick [2], [5,6], which considered a special type of $f$-structure with complementary frames related to a contact pair and called the assambley contact pair structure [4]. With these elements, they considered two almost complex structures and in case they are integrable, the contact pair structure is called normal. In [5] the authors describe this case and give necessary and sufficient conditions for a contact pair structure to be normal. Remark that the normality condition for different geometric structures is used in many papers, e.g., [15]. Basically, a contact pair consists of a pair of one-forms of constant and complementary classes such that each of them induces a contact form on the leaves of the characteristic foliation of the other. Similar notions were considered if instead of two one-forms, one takes a one-form and a closed two-form, respectively, two closed two-forms, satisfying certain conditions. In the first case, the structure is called contact-symplectic pair [1] and in the second one, symplectic pair [8].

Inspired by these considerations, we shall introduce the notion of (metric) paracontact pair structure and provide necessary and sufficient conditions for it to be normal. We shall also obtain the relations satisfied by the covariant derivatives of the fundamental form and of the endomorphism of the structure with respect to the Levi-Civita connection of the metric considered. The last section describes a 
construction performed on a principal $\mathbb{S}^{1}$-bundle which gives a metric paracontact pair structure on its total space.

A similar notion was defined by Bucki and Miernowski [11] under the name of $r$-paracontact structure, but they did not ask for the $r$ one-forms to be of constant and complementary classes. This last suplementary assumption implies certain properties of the leaves of the characteristic foliation.

\section{Definitions and Basic Properties}

The pair $\left(\eta_{1}, \eta_{2}\right)$ of one-forms is called contact pair of type $(h, k)$ on the $2(h+k+$ 1)-dimensional manifold $M$ [7] if

1. $\eta_{1} \wedge\left(\mathrm{d} \eta_{1}\right)^{h} \wedge \eta_{2} \wedge\left(\mathrm{d} \eta_{2}\right)^{k}$ is a volume form

2. $\left(\mathrm{d} \eta_{1}\right)^{h+1}=0$ and $\left(\mathrm{d} \eta_{2}\right)^{k+1}=0$.

To any contact pair $\left(\eta_{1}, \eta_{2}\right)$ can be associated two vector fields $\xi_{1}$ and $\xi_{2}$ called the Reeb vector fields of the contact pair, which are characterized by

1. $i_{\xi_{i}} \eta_{j}=\delta_{i j}$

2. $i_{\xi_{i}} \mathrm{~d} \eta_{j}=0, \quad$ for $\quad i, j \in\{1,2\}$.

Recall that a triple $(\varphi, \xi, \eta)$ consisting of a $(1,1)$-tensor field $\varphi$, a vector field $\xi$ and a one-form $\eta$ is called almost paracontact structure on the $(2 n+1)$-dimensional manifold $M[14]$ if

1. $\varphi^{2}=\mathrm{Id}-\eta \otimes \xi$

2. $\varphi \xi=0$

3. $\eta(\xi)=1$.

An almost paracontact structure $(\varphi, \xi, \eta)$ on $M$ is said to be normal if the induced almost product structure $E(X, Y):=(\varphi X+\eta(Y) \xi, \varphi Y+\eta(X) \xi), X, Y \in$ $\Gamma(T M)$, on the product manifold $M \times M$ is integrable, that is, its Nijenhuis tensor field

$$
\begin{aligned}
N_{E}((X, \bar{X}),(Y, \bar{Y})):=[E(X, \bar{X}), E(Y, \bar{Y})]+E^{2}[(X, \bar{X}),(Y, \bar{Y})] \\
-E[E(X, \bar{X}),(Y, \bar{Y})]-E[(X, \bar{X}), E(Y, \bar{Y})]
\end{aligned}
$$


vanishes for any $X, \bar{X}, Y, \bar{Y} \in \Gamma(T M)$. An equivalent condition is that the almost product structures $E_{1}:=\varphi-\eta \otimes \xi$ and $E_{2}:=\varphi+\eta \otimes \xi$ be integrable and satisfy $L_{\xi} \eta=0$, or that $N_{\varphi}(X, Y)-\mathrm{d} \eta(X, Y) \xi=0$, for any $X, Y \in \Gamma(T M)$, where

$$
N_{\varphi}(X, Y):=[\varphi X, \varphi Y]+\varphi^{2}[X, Y]-\varphi[\varphi X, Y]-\varphi[X, \varphi Y]
$$

and

$$
\mathrm{d} \eta(X, Y):=X(\eta(Y))-Y(\eta(X))-\eta([X, Y]) .
$$

Similarly like in the contact case, we introduce the notion of normal metric paracontact pair structure. Let $M$ be a $2(h+k+1)$-dimensional manifold.

Definition 1. The triple $\left(\eta_{1}, \eta_{2}, \varphi\right)$ is called paracontact pair structure if $\left(\eta_{1}, \eta_{2}\right)$ is a contact pair and $\varphi$ a $(1,1)$-tensor field such that

1. $\varphi^{2}=\mathrm{Id}-\eta_{1} \otimes \xi_{1}-\eta_{2} \otimes \xi_{2}$

2. $\varphi \xi_{1}=\varphi \xi_{2}=0$

where $\xi_{1}$ and $\xi_{2}$ are the Reeb vector fields of $\left(\eta_{1}, \eta_{2}\right)$.

From the definition follows $\eta_{i}(\varphi X)=0$, for any $X \in \Gamma(T M), i \in\{1,2\}$.

Example 2. Let $\left(\varphi_{1}, \xi_{1}, \eta_{1}\right)$ and $\left(\varphi_{2}, \xi_{2}, \eta_{2}\right)$ be two almost paracontact structures on the smooth manifold $M$. If $\eta_{i}\left(\xi_{j}\right)=\delta_{i j}$ and $\eta_{i} \circ \varphi_{j}=0, i, j \in\{1,2\}$, then $\left(\eta_{1}, \eta_{2}, \varphi_{ \pm}\right)$is a paracontact pair structure on the product manifold $M \times M$, where $\varphi_{ \pm}(X, Y):=\left(\varphi_{1} X \pm \eta_{1}(Y) \xi_{2}, \varphi_{2} Y \mp \eta_{2}(X) \xi_{1}\right), X, Y \in \Gamma(T M)$.

Definition 3. The quadruple $\left(\eta_{1}, \eta_{2}, \varphi, g\right)$ is called

1. almost metric paracontact pair structure if $\left(\eta_{1}, \eta_{2}, \varphi\right)$ is a paracontact pair structure and $g$ is a metric on $M$ such that

a) $i_{\xi_{i}} g=\eta_{i}, \quad i \in\{1,2\}$

b) $g(\varphi X, \varphi Y)=-g(X, Y)+\eta_{1}(X) \eta_{1}(Y)+\eta_{2}(X) \eta_{2}(Y), X, Y \in \Gamma(T M)$

where $\xi_{i}, i \in\{1,2\}$ are the Reeb vector fields of the contact pair $\left(\eta_{1}, \eta_{2}\right)$. In this case, we call g compatible metric

2. metric paracontact pair structure if $\left(\eta_{1}, \eta_{2}, \varphi\right)$ is a paracontact pair structure and $g$ is a metric on $M$ such that
a) $i_{\xi_{i}} g=\eta_{i}$
$i \in\{1,2\}$ 
b) $g(X, \varphi Y)=\left(\mathrm{d} \eta_{1}+\mathrm{d} \eta_{2}\right)(X, Y), \quad X, Y \in \Gamma(T M)$

where $\xi_{i}, i \in\{1,2\}$ are the Reeb vector fields of the contact pair $\left(\eta_{1}, \eta_{2}\right)$. In this case, we call g associated metric.

Remark that in both of these cases, $g\left(\xi_{i}, \xi_{j}\right)=\delta_{i j}, i, j \in\{1,2\}$ and $g(\varphi X, Y)=$ $-g(X, \varphi Y)$, for any $X, Y \in \Gamma(T M)$. If $g$ is associated metric, then it is also compatible, but conversely not.

Remark 4. Some authors take all the terms with opposite sign in the relation of compatibility of $g$ and some define metric paracontact structures as being almost paracontact structures with a compatible metric. We prefer our definitions to get an analogue of the notion of contact pair structure considered by Bande.

Proposition 5. Let $\left(\eta_{1}, \eta_{2}, \varphi\right)$ be a paracontact pair structure on the smooth manifold $M$. Then there exists a compatible metric $g$, so that $\left(\eta_{1}, \eta_{2}, \varphi, g\right)$ is an almost metric paracontact pair structure.

Proof: Like in the contact case [4], we can construct the compatible metric $g$ as follows: consider an arbitrary metric $g_{0}$ and set, for any $X, Y \in \Gamma(T M)$

$$
h(X, Y):=g_{0}\left(\varphi^{2} X, \varphi^{2} Y\right)+\eta_{1}(X) \eta_{1}(Y)+\eta_{2}(X) \eta_{2}(Y) .
$$

Then $g$ is defined by

$$
g(X, Y):=\frac{1}{2}\left[h(X, Y)-h(\varphi X, \varphi Y)+\eta_{1}(X) \eta_{1}(Y)+\eta_{2}(X) \eta_{2}(Y)\right] .
$$

Indeed, taking into account that $\varphi^{4}=\varphi^{2}$ and so $h\left(\varphi^{2} X, \varphi^{2} Y\right)=g_{0}\left(\varphi^{4} X, \varphi^{4} Y\right)=$ $g_{0}\left(\varphi^{2} X, \varphi^{2} Y\right)=h(X, Y)-\eta_{1}(X) \eta_{1}(Y)-\eta_{2}(X) \eta_{2}(Y)$, for any $X, Y \in \Gamma(T M)$, we obtain $g(\varphi X, \varphi Y)+g(X, Y)=\eta_{1}(X) \eta_{1}(Y)+\eta_{2}(X) \eta_{2}(Y)$, for any $X$, $Y \in \Gamma(T M)$.

To any contact pair $\left(\eta_{1}, \eta_{2}\right)$ of type $(h, k)$, one can associate the pairs of involutive distributions

1. $\mathcal{D}_{i}^{\prime}:=\operatorname{kerd} \eta_{i} \cap \operatorname{ker} \eta_{i}, i \in\{1,2\}$, whose foliations $\mathcal{F}_{1}^{\prime}$ and $\mathcal{F}_{2}^{\prime}$ are complementary and of codimension $2 h+1$ and $2 k+1$, respectively

2. $\mathcal{D}_{i}^{\prime \prime}:=\operatorname{kerd} \eta_{i} \cap \operatorname{ker} \eta_{1} \cap \operatorname{ker} \eta_{2}, i \in\{1,2\}$, whose foliations $\mathcal{F}_{1}^{\prime \prime}$ and $\mathcal{F}_{2}^{\prime \prime}$ are of codimension $2 h$ and $2 k$. 
Therefore, the tangent bundle can be decomposed in two ways

$$
T M=T \mathcal{F}_{1}^{\prime} \oplus T \mathcal{F}_{2}^{\prime}=T \mathcal{F}_{1}^{\prime \prime} \oplus T \mathcal{F}_{2}^{\prime \prime} \oplus\left\langle\xi_{1}\right\rangle \oplus\left\langle\xi_{2}\right\rangle
$$

and $T \mathcal{F}_{i}^{\prime}=T \mathcal{F}_{i}^{\prime \prime} \oplus\left\langle\xi_{j}\right\rangle, i \neq j$ and $\varphi$ is an almost product structure on $T \mathcal{F}_{1}^{\prime \prime} \oplus T \mathcal{F}_{2}^{\prime \prime}$. If the endomorphism $\varphi$ satisfies $\varphi\left(T \mathcal{F}_{i}^{\prime}\right) \subset T \mathcal{F}_{i}^{\prime}, i \in\{1,2\}$, we say that it is decomposable.

Proposition 6. Let $\left(\eta_{1}, \eta_{2}\right)$ be a contact pair on the smooth manifold $M$. Then there exist an endomorphism $\varphi$ of the tangent bundle and an associated metric $g$, so that $\left(\eta_{1}, \eta_{2}, \varphi, g\right)$ is a metric paracontact pair structure.

Proof: Like in the contact case [4], considering an arbitrary metric $g_{0}$, we obtain on $T \mathcal{F}_{1}^{\prime \prime} \oplus T \mathcal{F}_{2}^{\prime \prime}$ a metric $\bar{g}$ and an almost product structure $\bar{\varphi}$ compatible with $\bar{g}$. Then we define $\varphi:=\bar{\varphi}$ on $T \mathcal{F}_{1}^{\prime \prime} \oplus T \mathcal{F}_{2}^{\prime \prime}, \varphi \xi_{i}:=0$ and respectively, $g(X, Y):=\bar{g}(X, Y)$ on $T \mathcal{F}_{1}^{\prime \prime} \oplus T \mathcal{F}_{2}^{\prime \prime}, g\left(X, \xi_{i}\right):=\eta_{i}(X)$.

The following results have similar proofs as the corresponding results given in [6] for metric contact pairs.

Proposition 7. Let $\left(\eta_{1}, \eta_{2}, \varphi\right)$ be a paracontact pair structure on the smooth manifold $M$. Then there exists an associated metric $g$ for which the foliations $\mathcal{F}_{1}^{\prime}$ and $\mathcal{F}_{2}^{\prime}$ are g-orthogonal.

Proposition 8. Let $\left(\eta_{1}, \eta_{2}, \varphi, g\right)$ be a metric paracontact pair structure on the smooth manifold $M$.

1. The endomorphism $\varphi$ is decomposable if and only if the foliations $\mathcal{F}_{1}^{\prime}$ and $\mathcal{F}_{2}^{\prime}$ are g-orthogonal.

2. If the endomorphism $\varphi$ is decomposable, then the foliations $\mathcal{F}_{1}^{\prime}$ and $\mathcal{F}_{2}^{\prime}$ are minimal.

\section{Normal Metric Paracontact Pair Structures}

To a paracontact pair structure we associate two commuting almost product structures $\left(E_{1}, E_{2}\right)$ defined respectively, by

$$
E_{1}:=\varphi-\eta_{1} \otimes \xi_{2}-\eta_{2} \otimes \xi_{1}, \quad E_{2}:=\varphi+\eta_{1} \otimes \xi_{2}+\eta_{2} \otimes \xi_{1} .
$$

Using these almost product structures, we shall define the corresponding notion of integrability for paracontact pair structures. 
Definition 9. The paracontact pair structure $\left(\eta_{1}, \eta_{2}, \varphi\right)$ is called normal if the almost product structures $E_{1}$ and $E_{2}$ defined above, are integrable.

We will prove the following

Theorem 10. The necessary and sufficient condition for the paracontact pair structure $\left(\eta_{1}, \eta_{2}, \varphi\right)$ to be normal is to satisfy

$$
N_{\varphi}(X, Y)-\mathrm{d} \eta_{1}(X, Y) \xi_{1}-\mathrm{d} \eta_{2}(X, Y) \xi_{2}=0
$$

for any $X, Y \in \Gamma(T M)$, where $N_{\varphi}(X, Y):=[\varphi X, \varphi Y]+\varphi^{2}[X, Y]-\varphi[\varphi X, Y]$ $-\varphi[X, \varphi Y], X, Y \in \Gamma(T M)$.

Proof: The necessary and sufficient condition for the two almost product structures from the previous definition to be integrable is that their Nijenhuis tensor fields vanish. Computing the Nijenhuis tensor fields for $E_{1}$ and $E_{2}$ one gets, for any $X$, $Y \in \Gamma(T M)$

$$
\begin{aligned}
N_{E_{1}}(X, Y)= & N_{\varphi}(X, Y)-\left[\mathrm{d} \eta_{1}(X, Y)+\left(L_{\varphi X} \eta_{2}\right) Y-\left(L_{\varphi Y} \eta_{2}\right) X\right] \xi_{1} \\
& -\left[\mathrm{d} \eta_{2}(X, Y)+\left(L_{\varphi X} \eta_{1}\right) Y-\left(L_{\varphi Y} \eta_{1}\right) X\right] \xi_{2}-\eta_{1}(X)\left(L_{\xi_{2}} \varphi\right) Y \\
& -\eta_{2}(X)\left(L_{\xi_{1}} \varphi\right) Y+\eta_{1}(Y)\left(L_{\xi_{2}} \varphi\right) X+\eta_{2}(Y)\left(L_{\xi_{1}} \varphi\right) X
\end{aligned}
$$

and respectively

$$
\begin{aligned}
N_{E_{2}}(X, Y)= & N_{\varphi}(X, Y)-\left[\mathrm{d} \eta_{1}(X, Y)-\left(L_{\varphi X} \eta_{2}\right) Y+\left(L_{\varphi Y} \eta_{2}\right) X\right] \xi_{1} \\
& -\left[\mathrm{d} \eta_{2}(X, Y)-\left(L_{\varphi X} \eta_{1}\right) Y+\left(L_{\varphi Y} \eta_{1}\right) X\right] \xi_{2}+\eta_{1}(X)\left(L_{\xi_{2}} \varphi\right) Y \\
& +\eta_{2}(X)\left(L_{\xi_{1}} \varphi\right) Y-\eta_{1}(Y)\left(L_{\xi_{2}} \varphi\right) X-\eta_{2}(Y)\left(L_{\xi_{1}} \varphi\right) X
\end{aligned}
$$

where $N_{\varphi}$ and $\mathrm{d} \eta$ are given by (1) and (2), $\left(L_{X} \eta\right) Y:=X(\eta(Y))-\eta([X, Y])$ and $\left(L_{X} \varphi\right) Y:=[X, \varphi Y]-\varphi[X, Y], X, Y \in \Gamma(T M)$. But as $N_{E_{1}}$ and $N_{E_{2}}$ are zero if and only if their sum and their difference are zero, we obtain the following two equations

$$
\begin{gathered}
N_{\varphi}(X, Y)-\mathrm{d} \eta_{1}(X, Y) \xi_{1}-\mathrm{d} \eta_{2}(X, Y) \xi_{2}=0 \\
{\left[\left(L_{\varphi X} \eta_{2}\right) Y-\left(L_{\varphi Y} \eta_{2}\right) X\right] \xi_{1}+\left[\left(L_{\varphi X} \eta_{1}\right) Y-\left(L_{\varphi Y} \eta_{1}\right) X\right] \xi_{2}+\eta_{1}(X)\left(L_{\xi_{2}} \varphi\right) Y} \\
+\eta_{2}(X)\left(L_{\xi_{1}} \varphi\right) Y-\eta_{1}(Y)\left(L_{\xi_{2}} \varphi\right) X-\eta_{2}(Y)\left(L_{\xi_{1}} \varphi\right) X=0
\end{gathered}
$$

Notice that $\left(L_{\xi_{i}} \varphi\right) X \in \operatorname{ker} \eta_{j}$, for any $X \in \Gamma(T M), i, j \in\{1,2\}$. Indeed,

$$
\begin{aligned}
\eta_{j}\left(\left(L_{\xi_{i}} \varphi\right) X\right):=\eta_{j}\left(\left[\xi_{i}, \varphi X\right]\right)-\eta_{j}\left(\varphi\left[\xi_{i}, X\right]\right)=\eta_{j}\left(\left[\xi_{i}, \varphi X\right]\right) \\
=\xi_{i}\left(\eta_{j}(\varphi X)\right)-(\varphi X)\left(\eta_{j}\left(\xi_{i}\right)\right)-\mathrm{d} \eta_{j}\left(\xi_{i}, \varphi X\right)=0 .
\end{aligned}
$$


From the decomposition of $T M$ as the direct $\operatorname{sum} T M=T \mathcal{F}_{1}^{\prime \prime} \oplus T \mathcal{F}_{2}^{\prime \prime} \oplus\left\langle\xi_{1}\right\rangle \oplus\left\langle\xi_{2}\right\rangle$, we obtain an equivalent system with the previous one

$$
\begin{gathered}
N_{\varphi}(X, Y)-\mathrm{d} \eta_{1}(X, Y) \xi_{1}-\mathrm{d} \eta_{2}(X, Y) \xi_{2}=0 \\
\left(L_{\varphi X} \eta_{2}\right) Y-\left(L_{\varphi Y} \eta_{2}\right) X=0 \\
\left(L_{\varphi X} \eta_{1}\right) Y-\left(L_{\varphi Y} \eta_{1}\right) X=0 \\
\eta_{1}(X)\left(L_{\xi_{2}} \varphi\right) Y+\eta_{2}(X)\left(L_{\xi_{1}} \varphi\right) Y-\eta_{1}(Y)\left(L_{\xi_{2}} \varphi\right) X-\eta_{2}(Y)\left(L_{\xi_{1}} \varphi\right) X=0
\end{gathered}
$$

for any $X, Y \in \Gamma(T M)$.

The last three equations are implied by the first one. Indeed, taking $Y:=\xi_{i}$ in the first equation, we get

$$
\left[X, \xi_{i}\right]-\varphi\left[\varphi X, \xi_{i}\right]+\xi_{i}\left(\eta_{1}(X)\right) \xi_{1}+\xi_{i}\left(\eta_{2}(X)\right) \xi_{2}=0
$$

for any $X, Y \in \Gamma(T M)$. Then, for $X \mapsto \varphi X$

$$
\begin{aligned}
0=\left[\varphi X, \xi_{i}\right]-\varphi\left[\varphi^{2} X, \xi_{i}\right] & =-\left[\xi_{i}, \varphi X\right]+\varphi\left[\xi_{i}, X\right]+\varphi\left(\eta_{1}(X)\left[\xi_{1}, \xi_{i}\right]\right. \\
& \left.-\xi_{i}\left(\eta_{1}(X)\right) \xi_{1}\right)+\varphi\left(\eta_{2}(X)\left[\xi_{2}, \xi_{i}\right]-\xi_{i}\left(\eta_{2}(X)\right) \xi_{2}\right)=-\left(L_{\xi_{i}} \varphi\right) X
\end{aligned}
$$

If we apply $\eta_{i}$ to $N_{E_{1}}(\varphi X, Y)$ and consider the first equation of the system, we obtain

$$
\left(L_{\varphi^{2} X} \eta_{j}\right) Y-\left(L_{\varphi Y} \eta_{j}\right)(\varphi X)=0
$$

for any $X, Y \in \Gamma(T M)$, which is equivalent to

$$
\left(L_{X} \eta_{j}\right) Y-\left(L_{\eta_{1}(X) \xi_{1}+\eta_{2}(X) \xi_{2}} \eta_{j}\right) Y-\left(L_{\varphi Y} \eta_{j}\right)(\varphi X)=0
$$

for any $X, Y \in \Gamma(T M)$. For $X \mapsto \varphi X$, we get

$$
\left(L_{\varphi X} \eta_{j}\right) Y-\left(L_{\varphi Y} \eta_{j}\right) X+\left(L_{\varphi Y} \eta_{j}\right)\left(\eta_{1}(X) \xi_{1}+\eta_{2}(X) \xi_{2}\right)=0
$$

for any $X, Y \in \Gamma(T M)$ and the last term is zero because

$$
\begin{aligned}
\left(L_{\varphi Y} \eta_{j}\right)\left(\eta_{i}(X) \xi_{i}\right): & =(\varphi Y)\left(\eta_{i}(X) \delta_{i j}\right)-\eta_{j}\left(\left[\varphi Y, \eta_{i}(X) \xi_{i}\right]\right) \\
& =(\varphi Y)\left(\eta_{i}(X) \delta_{i j}\right)+\eta_{i}(X) \eta_{j}\left(\left[\xi_{i}, \varphi Y\right]\right)-(\varphi Y)\left(\eta_{i}(X)\right) \delta_{i j} \\
& =\eta_{i}(X)\left[\xi_{i}\left(\eta_{j}(\varphi Y)\right)-(\varphi Y)\left(\eta_{j}\left(\xi_{i}\right)\right)-\mathrm{d} \eta_{j}\left(\xi_{i}, \varphi Y\right)\right]=0 .
\end{aligned}
$$

Therefore, the last two equations of the system are also implied by the first one.

From relation (3) and the previous theorem, we deduce an equivalent condition for the normality of a paracontact pair structure 
Corollary 11. The paracontact pair structure $\left(\eta_{1}, \eta_{2}, \varphi\right)$ is normal if and only if

$$
\begin{gathered}
N_{\varphi}(X, Y)-\mathrm{d} \eta_{1}(X, Y) \xi_{1}=0, \text { for any } X, Y \in \Gamma\left(T \mathcal{F}_{2}^{\prime}\right) \\
N_{\varphi}(X, Y)-\mathrm{d} \eta_{2}(X, Y) \xi_{2}=0, \text { for any } X, Y \in \Gamma\left(T \mathcal{F}_{1}^{\prime}\right) \\
N_{\varphi}(X, Y)=0, \text { for any } X \in \Gamma\left(T \mathcal{F}_{1}^{\prime}\right), \quad Y \in \Gamma\left(T \mathcal{F}_{2}^{\prime}\right)
\end{gathered}
$$

where $N_{\varphi}(X, Y):=[\varphi X, \varphi Y]+\varphi^{2}[X, Y]-\varphi[\varphi X, Y]-\varphi[X, \varphi Y]$, for any $X, Y \in \Gamma(T M)$.

Corollary 11 implies

Lemma 12. If $\varphi$ is decomposable, the paracontact pair structure $\left(\eta_{1}, \eta_{2}, \varphi\right)$ is normal if and only if the induced contact forms and the structure tensor $\varphi$ are normal and $N_{\varphi}(X, Y)=0$, for any $X \in \Gamma\left(T \mathcal{F}_{1}^{\prime}\right), Y \in \Gamma\left(T \mathcal{F}_{2}^{\prime}\right)$.

The following result is similar to one given in [12] for product manifolds.

Theorem 13. Let $\left(M_{1}, \varphi_{1}, \xi_{1}, \eta_{1}\right)$ and $\left(M_{2}, \varphi_{2}, \xi_{2}, \eta_{2}\right)$ be almost paracontact manifolds. Then the paracontact pair structure $\left(\eta_{1}, \eta_{2}, \varphi_{1} \oplus \varphi_{2}\right)$ on $M_{1} \times M_{2}$ is normal if and only if $\left(\varphi_{1}, \xi_{1}, \eta_{1}\right)$ and $\left(\varphi_{2}, \xi_{2}, \eta_{2}\right)$ are normal as almost paracontact structures.

Proof: The first implication is true due to Corollary 11 and the converse one, by Lemma 12.

Consider a metric paracontact pair structure $\left(\eta_{1}, \eta_{2}, \varphi, g\right)$ and put $\omega(X, Y):=$ $g(X, \varphi Y)$. Then $\omega$ is exact, more precisely, $\omega=\mathrm{d} \eta_{1}+\mathrm{d} \eta_{2}$.

In the following propositions, we shall underline some properties of the covariant derivatives of $\omega$ and $\varphi$ with respect to the Levi-Civita connection $\nabla$ of $g$.

From $\mathrm{d} \omega=0$ we get

$$
\left(\nabla_{X} \omega\right)(Y, Z)+\left(\nabla_{Y} \omega\right)(Z, X)+\left(\nabla_{Z} \omega\right)(X, Y)=0
$$

for any $X, Y, Z \in \Gamma(T M)$.

Proposition 14. Let $\left(\eta_{1}, \eta_{2}, \varphi, g\right)$ be a metric paracontact pair structure on the smooth manifold $M$ and denote by $\nabla$ the Levi-Civita connection associated to $g$. Then, for any $X, Y, Z \in \Gamma(T M)$, one has

1.

$$
\begin{aligned}
& \left(\nabla_{X} \omega\right)(\varphi Y, \varphi Z)-\left(\nabla_{X} \omega\right)(Y, Z)=\eta_{1}(Y)\left(\nabla_{X} \eta_{1}\right)(\varphi Z) \\
+ & \eta_{2}(Y)\left(\nabla_{X} \eta_{2}\right)(\varphi Z)-\eta_{1}(Z)\left(\nabla_{X} \eta_{1}\right)(\varphi Y)-\eta_{2}(Z)\left(\nabla_{X} \eta_{2}\right)(\varphi Y)
\end{aligned}
$$


2.

$$
\begin{array}{r}
\left(\nabla_{X} \omega\right)(\varphi Y, Z)-\left(\nabla_{X} \omega\right)(Y, \varphi Z)=\eta_{1}(Y)\left(\nabla_{X} \eta_{1}\right) Z+\eta_{2}(Y)\left(\nabla_{X} \eta_{2}\right) Z \\
+\eta_{1}(Z)\left(\nabla_{X} \eta_{1}\right) Y+\eta_{2}(Z)\left(\nabla_{X} \eta_{2}\right) Y
\end{array}
$$

3.

$$
\begin{aligned}
\left(\nabla_{X} \omega\right)(Z, Y)-\left(\nabla_{\varphi X} \omega\right)(\varphi Z, Y)=- & \frac{1}{2} \eta_{1}(Z)\left(L_{\xi_{1}} g\right)(Y, \varphi X) \\
& -\frac{1}{2} \eta_{2}(Z)\left(L_{\xi_{2}} g\right)(Y, \varphi X) .
\end{aligned}
$$

Proof: The first two relations follow by a direct computation. Writing the relation (12) for circular permutations $-(X, \varphi Z, \varphi Y)+(Y, \varphi X, \varphi Z)+(Z, \varphi Y, \varphi X)-$ $(X, Z, Y)$ and taking into account that $\left(L_{\xi_{i}} g\right)(X, Y)=\left(\nabla_{X} \eta_{i}\right) Y+\left(\nabla_{Y} \eta_{i}\right) X$, $i \in\{1,2\}$, we obtain the last relation.

In particular, if we put $X=\xi_{i}$ in (15), we get $\nabla_{\xi_{i}} \omega=0, i \in\{1,2\}$.

Proposition 15. Let $\left(\eta_{1}, \eta_{2}, \varphi, g\right)$ be a metric paracontact pair structure on the smooth manifold $M$ and denote by $\nabla$ the Levi-Civita connection associated to $g$. Then, for any $X, Y, Z \in \Gamma(T M)$, one has

$$
\begin{gathered}
2 g\left(\left(\nabla_{X} \varphi\right) Y, Z\right)=-g\left(N_{\varphi}(Y, Z), \varphi X\right) \\
+\eta_{1}(X)\left[\left(L_{\varphi Y} \eta_{1}\right) Z-\left(L_{\varphi Z} \eta_{1}\right) Y\right]+\eta_{2}(X)\left[\left(L_{\varphi Y} \eta_{2}\right) Z-\left(L_{\varphi Z} \eta_{2}\right) Y\right] \\
-\eta_{1}(Y) \mathrm{d} \eta_{1}(\varphi Z, X)-\eta_{2}(Y) \mathrm{d} \eta_{2}(\varphi Z, X)+\eta_{1}(Z) \mathrm{d} \eta_{1}(\varphi Y, X)+\eta_{2}(Z) \mathrm{d} \eta_{2}(\varphi Y, X) .
\end{gathered}
$$

Moreover, if $\left(\eta_{1}, \eta_{2}, \varphi, g\right)$ is normal, then the relation above becomes

$$
\begin{aligned}
2 g\left(\left(\nabla_{X} \varphi\right) Y, Z\right)= & -\eta_{1}(Y) \mathrm{d} \eta_{1}(\varphi Z, X)-\eta_{2}(Y) \mathrm{d} \eta_{2}(\varphi Z, X) \\
& +\eta_{1}(Z) \mathrm{d} \eta_{1}(\varphi Y, X)+\eta_{2}(Z) \mathrm{d} \eta_{2}(\varphi Y, X)
\end{aligned}
$$

for any $X, Y, Z \in \Gamma(T M)$.

Proof: Considering the usual relations satisfied by the Levi-Civita connection $\nabla$ and computing all the terms in the equation, we easily get the first relation. For the second one, notice that $g\left(N_{\varphi}(Y, Z), \varphi X\right)=0$, for any $X, Y, Z \in \Gamma(T M)$, $\omega$ is closed and the last two terms are zero from the computations in the proof of Theorem 10.

Remark 16. In [7] Bande and Kotschick showed a relation between normal metric contact pairs and Vaisman metrics [9], [13]. 
An analogue of the construction described by Bande and Hadjar [5] performed on a Boothby-Wang fibration can be done in our case. Here, the base manifold $B$ is assumed to carry a paracontact-symplectic pair structure $(\eta, \omega, \psi)$ of type $(h, k)$, that is [1] a triple consisting of a one-form $\eta$, a closed two-form $\omega$ and an endomorphism $\psi$ of the tangent bundle satisfying

1. $\eta \wedge(\mathrm{d} \eta)^{h} \wedge \omega^{k}$ is a volume form

2. $(\mathrm{d} \eta)^{h+1}=0$ and $\omega^{k+1}=0$

3. $\psi^{2}=\mathrm{Id}-\eta \otimes \xi$

4. $\psi \xi=0$

where $\xi$ is the Reeb vector field of the pair $(\eta, \omega)$, uniquely defined by $i_{\xi} \eta=1$ and $i_{\xi} \mathrm{d} \eta=i_{\xi} \omega=0$. Moreover, if a metric $h$ on $B$ satisfies

1. $i_{\xi} h=\eta$

2. $h(X, \psi Y)=(\mathrm{d} \eta+\omega)(X, Y), X, Y \in \Gamma(T B)$

then $(\eta, \omega, \psi, h)$ is called metric paracontact-symplectic pair structure. Given a paracontact-symplectic pair structure, such a metric always exists, its construction being similar as in the case of paracontact pair structures. Under certain assumptions, a metric paracontact-symplectic pair structure can be lifted from the base space of a principal $\mathbb{S}^{1}$-bundle to a metric paracontact pair structure on the total space. Precisely, we have

Theorem 17. Let $\pi: M \rightarrow B$ be a principal $\mathbb{S}^{1}$-bundle and $(\eta, \omega, \psi, h)$ a metric paracontact-symplectic pair structure. Assume that $B$ is closed and $[\omega] \in$ $H^{2}(B, \mathbb{Z})$ is an integral cohomology class. Then the total space $M$ carries an $\mathbb{S}^{1}$-invariant metric paracontact pair structure.

Proof: Denote by $X^{*}$ the horizontal lift of a vector field $X$ on $B$ and define the metric paracontact pair structure $\left(\eta_{1}, \eta_{2}, \varphi, g\right)$ on $M$ as follows: take $\eta_{1}$ the connection form of the bundle $\left(\mathrm{d} \eta_{1}=\pi^{*} \omega\right)$, which is well defined as $\omega$ represents an integral cohomology class, $\eta_{2}:=\pi^{*} \eta, \varphi X:=\left[\psi\left(\pi_{*}(X)\right)\right]^{*}, X \in \Gamma(T M)$ and $g:=\pi^{*} h+\eta_{1} \otimes \eta_{1}+\eta_{2} \otimes \eta_{2}$. The Reeb vector fields of the pair $\left(\eta_{1}, \eta_{2}\right)$ satisfy: $\xi_{1}$ is tangent to the action and $\xi_{2}=\xi^{*}$. The endomorphism $\varphi$ satisfies

$$
\begin{aligned}
\varphi^{2} X & =\left[\psi\left(\psi\left(\pi_{*}(X)\right)\right)\right]^{*}=\left[\pi_{*}(X)\right]^{*}-\left[\eta\left(\pi_{*}(X)\right) \xi\right]^{*} \\
& =X-\eta_{1}(X) \xi_{1}-\pi^{*}\left(\eta\left(\pi_{*}(X)\right)\right) \xi^{*}=X-\eta_{1}(X) \xi_{1}-\left(\pi^{*} \eta\right)(X) \xi^{*} \\
& =X-\eta_{1}(X) \xi_{1}-\eta_{2}(X) \xi_{2}
\end{aligned}
$$


for any $X \in \Gamma(T M)$ and the metric $g$ is associated to the paracontact pair structure $\left(\eta_{1}, \eta_{2}, \varphi\right)$

$$
\begin{aligned}
g(X, \varphi Y) & =\left(\pi^{*} h\right)(X, \varphi Y)=h\left(\pi_{*}(X), \psi\left(\pi_{*}(Y)\right)\right) \circ \pi \\
& =\left[(\mathrm{d} \eta+\omega)\left(\pi_{*}(X), \pi_{*}(Y)\right)\right] \circ \pi=\left[\pi^{*}(\mathrm{~d} \eta)+\pi^{*} \omega\right](X, Y) \\
& =\left[\mathrm{d}\left(\pi^{*} \eta\right)+\mathrm{d} \eta_{1}\right](X, Y)=\left(\mathrm{d} \eta_{1}+\mathrm{d} \eta_{2}\right)(X, Y)
\end{aligned}
$$

for any $X, Y \in \Gamma(T M)$.

Remark 18. Similar results were proved by Bande and Kotschick [8] if the base space carries a symplectic pair $\left(\omega_{1}, \omega_{2}\right)$ or a contact-symplectic pair $(\eta, \omega)$ and Bande and Hadjar [5] made this construction for contact-symplectic pair structures $(\eta, \omega, \psi)$.

\section{Acknowledgements}

The author acknowledges the support by the research grant PN-II-ID-PCE-2011-30921. She wants to express also her thanks to the referees for the valuable remarks and suggestions which definetely have improved the paper.

\section{References}

[1] Bande G., Formes de Contact Généralisé, Couples de Contact et Couples Contacto-Symplectiques, Thèse de Doctorat, Université de Haute Alsace, Mulhouse 2000.

[2] Bande G., Ghiggini P. and Kotschick D., A Stability Theorem for Contact and Symplectic Pairs, Int. Math. Res. Not. 68 (2004) 3673-3688.

[3] Bande G. and Hadjar A., Contact Pairs, Tohoku Math. J. 57 (2005) 247-260.

[4] Bande G. and Hadjar A., Contact Pair Structures and Associated Metrics, In: Proceedings of the VIII International Colloquium on Diff. Geom., World Scientific, Singapore 2009, pp. 266-275.

[5] Bande G. and Hadjar A., On Normal Contact Pairs, Int. J. Math. 21 (2010) 737-754.

[6] Bande G. and Hadjar A., On the Characteristic Foliations of Metric Contact Pairs, arXiv:1003.0281v2/math.DG (2010).

[7] Bande G. and Kotschick D., Contact Pairs and Locally Conformally Symplectic Structures, http://129.187.111.185/ dieter/lcs-cp.pdf. 
[8] Bande G. and Kotschick D., The Geometry of Symplectic Pairs, Trans. Amer. Math. Soc. 358 (2006) 1643-1655.

[9] Belgun F., On the Metric Structure of Non-Kähler Complex Surfaces, Math. Ann. 317 (2000) 1-40.

[10] Blair D., Ludden G. and Yano K., Geometry of Complex Manifolds Similar to Calabi-Eckmann Manifolds, J. Diff. Geom. 9 (1974) 263-274.

[11] Bucki A. and Miernowski A., Almost r-Paracontact Structures, Ann. Univ. Mariae Curie-Sklodowska Sect. A 39 (1985) 13-26.

[12] Morimoto A., On Normal Almost Contact Structures, J. Math. Soc. Japan 15 (1963) 420-436.

[13] Ornea L. and Verbitsky M., Structure Theorem for Compact Vaisman Manifolds, Math. Research Letters 10 (2003) 799-805.

[14] Sato I., On a Structure Similar to Almost Contact Structure, Tensor N. S. 30 (1976) 219-224.

[15] Vaisman I., From Generalized Kähler to Generalized Sasakian Structures, J. Geom. Symmertry Phys. 18 (2010) 63-86.

Adara M. Blaga

Department of Mathematics

West University of Timişoara

Bld. V. Pârvan nr. 4

300223 Timişoara, ROMÂNIA

E-mail address: adara@math.uvt.ro 